Die Cartesischen Produkte und die Multiplikation von Maßfunktionen. Ir. 233

Bemerkung: Den Satz von FuBIN kann man auch für summierbare Ortsfunktionen über ein beliebiges Element $\mathfrak{a} \in \bar{L}$ formulieren: denn man kann immer eine Ortsfunktion $f^{*}$ auf $\mathfrak{e}=e_{1} \times e_{3}{ }^{30}$ ) erklären, welche summierbar über e für $\varphi$ ist und, als Ortsfunktion auf a betrachtet, gleich $f$ ist, dagegen auf e-a gleich Null ist.

${ }^{30}$ Hier bedeutet e das volle Element von $\bar{L}$, welches wegen der Vollständigkeit von $R_{1}$ bzw. $R_{\mathrm{z}}$ existiert.

(Eingegangen am 20. Februar 1948.)

\title{
Berichtigungen zum ersten Teil dieser Arbeit.
}

[Math. Annalen 120, S. 43-74 (1947)].

Seite 46, Zeile 20 von unten, Ax. $6^{*}$. lies , $x \odot(a+b)^{*}$ statt , $a \odot(a+b) *$

Seite 64, Zeile 17 von oben, Formel (1): lies, , $\mathfrak{a}^{(1)} \supseteq \mathfrak{a}^{(2)} \supseteq \cdots \cdots$ statt , $\mathfrak{a}^{(1)} \leqq a^{(2)} \leqq \cdots *$.

Seite 69, Zeile 5 von unten ohne FuBnote: lies , $0=\lim \left[\varphi\left(v_{t}\right)-\varphi(a)\right]=\lim \varphi\left(v_{k}+a\right)^{c k}$ statt, $\lim _{k \rightarrow \infty} \varphi\left(v_{k}\right)=\varphi(a)^{*}$.

Seite 70 , Zeile 3 und, 5 von oben: lies , $\varphi\left(v_{k}+a_{v_{k}}\right)^{*}$ statt,$\varphi\left(v_{k}+a_{k}\right)^{*}$.

Seite 70, Zeile 10-11 von unten: streiche ,d. h. $\lim \varphi\left(a_{m}\right)=\varphi(a)^{*}$.

Seite 71, Zeile 9 von aben, zweite Zeile von Satz 15: lies, $\phi$-totalkonvergiert"s statt, "$\varphi$-konvergiert".

Seite 71, Zeile 10 von unten, zweite Zeile von Satz 16: streiche ,lim $\varphi\left(a_{n}\right)=\varphi(a)$, also".

Seite 71, Zeile 4 und 5 von unten: lies

$$
, \varphi-\lim v_{n}=\varphi-\lim d_{n}=a .
$$

Nun ist $d_{n} \subseteq a_{n} \subseteq w_{n}$, also $\varphi \cdot \lim a_{n}=a_{*} *$

Statt

$$
, \lim \varphi\left(v_{n}\right)=\lim \varphi\left(d_{n}\right)=\varphi(a)
$$

Nun ist $d_{n} \subseteq a_{n} \leqq v_{n}$ also $\varphi\left(d_{n}\right) \leqq \varphi\left(a_{n}\right) \leqq \varphi\left(v_{n}\right)$, d. h. $\lim \varphi\left(a_{n}\right)=\varphi(a)$.* 